SUNY-NTG-98/25

\title{
From chiral Random Matrix Theory
}

\section{to chiral Perturbation Theory}

\author{
J.C. Osborn, D. Toublan and J.J.M. Verbaarschot \\ Department of Physics and Astronomy, SUNY, Stony Brook, New York 11794
}

\begin{abstract}
We study the spectrum of the QCD Dirac operator by means of the valence quark mass dependence of the chiral condensate in partially quenched Chiral Perturbation Theory (pqChPT) in the supersymmetric formulation of Bernard and Golterman. We consider valence quark masses both in the ergodic domain $\left(m_{v} \ll E_{c}\right)$ and the diffusive domain $\left(m_{v} \gg E_{c}\right)$. These domains are separated by a mass scale $E_{c} \sim F^{2} / \Sigma_{0} L^{2}$ (with $F$ the pion decay constant, $\Sigma_{0}$ the chiral condensate and $L$ the size of the box). In the ergodic domain the effective super-Lagrangian reproduces the microscopic spectral density of chiral Random Matrix Theory (chRMT). We obtain a natural explanation of Damgaard's relation between the spectral density and the finite volume partition function with two additional flavors. We argue that in the ergodic domain the natural measure for the superunitary integration in the pqChPT partition function is noncompact. We find that the tail of the two-point spectral correlation function derived from pqChPT agrees with the chRMT result in the ergodic domain. In the diffusive domain we extend the results for the slope of the Dirac spectrum first obtained by Smilga and Stern. We find that the spectral density diverges logarithmically for nonzero topological susceptibility. We study the transition between the ergodic and the diffusive domain and identify a range where chRMT and pqChPT coincide.
\end{abstract}

PACS: 11.30.Rd, 12.39.Fe, 12.38.Lg, 71.30.+h// / noindent Keywords: QCD Dirac operator; Chiral random matrix theory; Partially quenched chiral perturbation theory; Thouless energy; Microscopic spectral density; Valence quark mass dependence 


\section{Introduction}

Spontaneous breaking of chiral symmetry is believed to be an important property of the strong interaction. In the limit of massless quarks the QCD Lagrangian is invariant under $U_{L}\left(N_{f}\right) \times U_{R}\left(N_{f}\right)$ transformations, but the vacuum state is not. The analysis of hadron spectra and numerical simulations [1, 2, 3] on the lattice strongly support this assertion. The order parameter for spontaneous breaking of chiral symmetry is the chiral condensate. In the chiral limit it is related to the spectral density of the QCD Dirac operator by means of the Banks-Casher formula [4],

$$
\Sigma_{0}=\lim \frac{\pi \rho(0)}{V}
$$

where $V$ is the volume of space-time and the spectral density is defined by

$$
\rho(\lambda)=\sum_{k} \delta\left(\lambda-\lambda_{k}\right)
$$

Here, the $\lambda_{k}$ are the eigenvalues of the Euclidean QCD Dirac operator. They will be called virtualities in this paper. In (1) the thermodynamic limit is taken before the chiral limit. For broken chiral symmetry, the eigenvalues near zero are spaced as $1 / \rho(0)=\pi / \Sigma_{0} V$. In order to study the behavior of the Dirac spectrum in the approach to the thermodynamic limit it is therefore natural to introduce the microscopic variable [5]

$$
u=V \lambda \Sigma_{0}
$$

and the microscopic spectral density [5] defined by

$$
\rho_{S}(u)=\lim _{V \rightarrow \infty} \frac{1}{V \Sigma_{0}}\left\langle\rho\left(\frac{u}{V \Sigma_{0}}\right)\right\rangle .
$$

In general, the average $\langle\cdots\rangle$ in this equation is over the probability distribution of the eigenvalues. It was conjectured in [5] that the microscopic spectral density is universal and is given by chiral Random Matrix Theories (chRMT) with the global symmetries of the QCD partition function. This conjecture has been verified both by instanton liquid [6] and by lattice QCD [7, 8, 9, 10] simulations. It was also found from lattice QCD simulations that eigenvalue correlations on the microscopic scale, both near zero virtuality and in the bulk of the spectrum, are given by Random Matrix Theory [11, 12, 13, 9]. The foundation

for these observations was laid by universality studies [14, 15, 16, 17, 18, 19, 20, 21] in which it was shown that the microscopic spectral density and other spectral correlations on the scale of individual level spacings are not affected by macroscopic deformations of the random matrix ensemble. The interpretation is that quarks undergo a chaotic/diffusive motion in four Euclidean dimensions and one artificial time dimension. Such picture is also suggested by the success of microcanonical lattice QCD simulations [22]. According 
to the Bohigas conjecture [23] the correlations of the eigenvalues on the microscopic scale are then given by Random Matrix Theory. In Ref. [24 chiral symmetry breaking was also related to transport properties of quarks in $4+1$ dimensions.

Of course, the dynamics of QCD, which is not included in chRMT, should enter in the Dirac spectrum. Therefore, we expect a scale above which the microscopic spectral density and the spectral correlations are not given by chRMT anymore. The existence of such scale is well known in the context of mesoscopic physics (see 25, 26, 27 for reviews). It is known as the Thouless energy, which is the inverse diffusion time of an electron through the sample 28]

$$
E_{c}=\frac{\hbar D}{L^{2}},
$$

where $D$ is the diffusion constant and $L$ is the linear dimension of the box. In the case of QCD, the diffusion process is that of a quark propagating through the Yang-Mills fields in a $4+1$ dimensional space time. A second energy scale that enters in mesoscopic physics is $\hbar / \tau_{e}$, where $\tau_{e}$ is the elastic diffusion time. Based one these two scales three different domains for the energy difference, $\delta E$, that enters in the two-point correlation function, can be distinguished: the ergodic domain, the diffusive domain or AltshulerShklovskii domain and the ballistic domain. For energy differences $\delta E \ll E_{c}$ eigenvalue correlations are given by RMT. Since for time scales larger than the diffusion time, an initially localized wave packet explores the complete phase space, this regime is known as the ergodic regime. In the diffusive regime, defined by $E_{c} \ll \delta E \ll \hbar / \tau_{e}$ only part of the phase space is explored by an initially localized wave packet, resulting in the disappearance of eigenvalue correlations. In this paper we don't consider the ballistic regime with $\delta E \gg \hbar / \tau_{e}$. For an interpretation of the Thouless energy in terms of the spreading width we refer to [29, 30]. As was shown in 31, 32], the spectral two-point function can be related to the semiclassical return probability which provides a simple intuitive picture of its asymptotic behavior. For other recent studies on this topic we refer to 33 , 34, 35, 36, 37, 38, 39. What has emerged from these studies is that there is a close relation between eigenvalue correlations and localization properties of the wave functions. The interpretation of spontaneous chiral symmetry breaking as a delocalization transition was made earlier in [40]. By analogy with the Kubo formula, $\Sigma_{0}$ plays the role of the conductivity [40].

In QCD Dirac spectra the Thouless energy was identified as the scale where the inverse pion mass is of the order of the linear size of the box [41, 42]. Using standard relations based on the approximate chiral symmetry of the QCD partition function it has been suggested that 41, 43, 42,

$$
E_{c} \sim \frac{F^{2}}{\Sigma_{0} L^{2}}
$$


where $F$ is the pion decay constant. The existence of such a scale has been observed in instanton liquid [41, 43] and lattice QCD [8, 9] simulations. One of the main goals of this paper is to identify this scale by means of partially quenched Chiral Perturbation Theory 45.

In this paper we will discuss four different partition functions: the Euclidean QCD partition function, the effective finite volume partition function [46, 47], the chiral random matrix theory partition function [5] and the partition function for partially quenched Chiral Perturbation Theory [45]. Below, we briefly define the first three partition functions whereas the last one is discussed extensively in the remainder of this paper. At this point we only mention that pqChPT is an effective mesonic theory which is generally believed to describe the low-energy limit of QCD. In addition to the usual $N_{f}$ sea quarks, this theory has $k$ (in this paper $k=1$ ) valence quarks and an equal number of bosonic ghost quarks with the same mass $m_{v}$. Without a source term that breaks the supersymmetry the valence sector drops out of the partition function. In other words, the valence quark mass only appears in the operators.

The Euclidean QCD partition function with fundamental quarks is given by

$$
Z_{Q C D}(\mathcal{M})=\int d A \operatorname{det}(\not D+\mathcal{M}) e^{-S_{Y M}}
$$

where $\mathcal{M}$ is the quark mass matrix, $\not D$ is the Euclidean Dirac operator and $S_{Y M}$ is the Yang-Mills action. The integral is over all gauge field configurations $A$. The eigenvalues of the Dirac operator are defined by

$$
\not D \psi_{k}=i \lambda_{k} \psi_{k}
$$

Notice that for $\lambda_{k} \neq 0$ the eigenfunctions occur in pairs $\psi_{k}, \gamma_{5} \psi_{k}$ with opposite eigenvalues. The quark mass dependence resides in the determinant.

For an Euclidean space-time volume with linear dimension in the range

$$
1 / \Lambda \ll L \ll 1 / m_{\pi}
$$

( $\Lambda$ is a typical hadronic scale and $m_{\pi}$ is the pion mass) the mass dependence of the QCD partition function is given by the effective finite volume partition function. For $N_{f}$ flavors, vacuum angle $\theta$ and mass matrix $\mathcal{M}$ it is defined by [46, 47]

$$
Z_{N_{f}}^{\mathrm{eff}}(\mathcal{M}, \theta) \sim \int_{U \in G / H} d U e^{V \Sigma_{0} \operatorname{Re} \operatorname{Tr} \mathcal{M} U e^{i \theta / N_{f}}} .
$$

The integral is over the Goldstone manifold associated with chiral symmetry breaking from $G$ to $H$. For three or more colors with fundamental fermions $G / H=S U_{L}\left(N_{f}\right) \times$ $S U_{R}\left(N_{f}\right) / S U_{A}\left(N_{f}\right)$. The effective finite volume partition function in the sector of topological charge $\nu$ follows by Fourier inversion with respect to vacuum angle $\theta$. The partition 
function for $\nu=0$ is thus given by (10) with the integration over $S U\left(N_{f}\right)$ replaced by an integral over $U\left(N_{f}\right)$. For an elaborate discussion of this partition function we refer to 47.

The chiral random matrix partition function with the global symmetries of the QCD partition function as input is defined by [5, 48]

$$
Z_{N_{f}, \nu}^{\beta}\left(m_{1}, \cdots, m_{N_{f}}\right)=\int D W \prod_{f=1}^{N_{f}} \operatorname{det}\left(\mathcal{D}+m_{f}\right) e^{-\frac{N \beta}{4} \Sigma \operatorname{Tr} W^{\dagger} W}
$$

where

$$
\mathcal{D}=\left(\begin{array}{cc}
0 & i W \\
i W^{\dagger} & 0
\end{array}\right)
$$

and $W$ is a $n \times m$ matrix with $\nu=|n-m|$ and $N=n+m$. As is the case in QCD, we assume that the equivalent of the topological charge $\nu$ does not exceed $\sqrt{N}$, so that, to a good approximation, $n=N / 2$. Then the parameter $\Sigma$ can be identified as the chiral condensate and $N$ as the dimensionless volume of space time (Our units are defined such that the density of the modes $N / V=1)$. The matrix elements of $W$ are either real $(\beta=1$, chiral Gaussian Orthogonal Ensemble (chGOE)), complex $(\beta=2$, chiral Gaussian Unitary Ensemble (chGUE)), or quaternion real $(\beta=4$, chiral Gaussian Symplectic Ensemble (chGSE)). For QCD with three or more colors and quarks in the fundamental representation the matrix elements of the Dirac operator are complex and we have $\beta=2$. It can be shown that in the domain (9) the random matrix partition function can be mapped onto the effective finite volume partition function [5]. For more discussion of the chRMT partition function we refer to 49.

In this paper we study the Dirac spectrum by means of the valence quark mass dependence of the chiral condensate [50, 51, 52]

$$
\Sigma\left(m_{v}\right)=\frac{1}{V} \sum_{k}\left\langle\frac{1}{m_{v}+i \lambda_{k}}\right\rangle
$$

where $\langle\cdots\rangle$ denotes an average with respect to the distribution of the eigenvalues. The spectral density follows from the discontinuity across the imaginary axis,

$$
\left.\operatorname{Disc}\right|_{m_{v}=i \lambda} \Sigma\left(m_{v}\right)=\lim _{\epsilon \rightarrow 0} \Sigma(i \lambda+\epsilon)-\Sigma(i \lambda-\epsilon)=2 \pi \sum_{k}\left\langle\delta\left(\lambda+\lambda_{k}\right)\right\rangle=2 \pi\langle\rho(\lambda)\rangle .
$$

The two point spectral correlation function is given by

$$
\left\langle\rho(\lambda) \rho\left(\lambda^{\prime}\right)\right\rangle=\left.\frac{1}{4 \pi^{2}} \operatorname{Disc}\right|_{m_{v}=i \lambda, m_{v^{\prime}}=i \lambda^{\prime}} \sum_{k, l}\left\langle\frac{1}{i \lambda_{k}+m_{v}} \frac{1}{i \lambda_{l}+m_{v^{\prime}}}\right\rangle .
$$


On the other hand, the valence quark mass dependence can be calculated from the partially quenched partition function. By differentiating with respect to the valence quark masses we obtain in the parametrization discussed in section 2 ,

$$
\left\langle\rho(\lambda) \rho\left(\lambda^{\prime}\right)\right\rangle^{C}=\left.\frac{1}{8 \pi^{2}} \operatorname{Disc}\right|_{m_{v}=i \lambda, m_{v^{\prime}}=i \lambda^{\prime}} \frac{\Sigma_{0}^{2}}{F_{\pi}^{4}} \int d^{4} x \int d^{4} y\left\langle\xi_{v v^{\prime}}(x) \xi_{v v^{\prime}}(x) \xi_{v v^{\prime}}(y) \xi_{v v^{\prime}}(y)\right\rangle_{p q}^{C}
$$

where the superscript $C$ refers to the connected correlation function which is defined as $\left\langle\rho\left(\lambda_{1}\right) \rho\left(\lambda_{2}\right)\right\rangle^{C}=\left\langle\rho\left(\lambda_{1}\right) \rho\left(\lambda_{2}\right)\right\rangle-\left\langle\rho\left(\lambda_{1}\right)\right\rangle\left\langle\rho\left(\lambda_{2}\right)\right\rangle$. The notation $\langle\cdots\rangle_{p q}$ denotes averaging with respect to the partially quenched partition function. The meson fields with quark content corresponding to a mass $M=\left(m_{v}+m_{v^{\prime}}\right) \Sigma_{0} / F^{2}$ are denoted by $\xi_{v v^{\prime}}$. The expectation value of the product of the meson fields can be expressed in terms of the meson propagator in momentum space. Taking into account the combinatorial factors and performing the integrals over $x$ and $y$ and one momentum integral this results in

$$
\left\langle\rho(\lambda) \rho\left(\lambda^{\prime}\right)\right\rangle^{C}=\left.\frac{1}{4 \pi^{2}} \frac{\Sigma_{0}^{2}}{F^{4}} \operatorname{Disc}\right|_{m_{v}=i \lambda, m_{v^{\prime}}=i \lambda^{\prime}} \sum_{q} \frac{1}{\left(q^{2}+M^{2}\right)^{2}} .
$$

We have expressed the two-point correlation function as a sum over momentum modes. Such relations are well known from the theory of disordered systems (see for example [33, 31, 32]). The zero momentum modes reproduce the asymptotic limit of the random matrix result for the two-point correlation function. This can be seen by evaluating the discontinuities after substitution of the Gell-Mann-Oakes-Renner relation for the meson mass. For $\left|\lambda-\lambda^{\prime}\right| \ll \lambda$ but much larger than the average level spacing we obtain

$$
\left\langle\rho(\lambda) \rho\left(\lambda^{\prime}\right)\right\rangle^{C} \sim-\frac{1}{2 \pi^{2}} \frac{1}{\left(\lambda-\lambda^{\prime}\right)^{2}} .
$$

which is the well-known random matrix result. The full correlation function can only be obtained by means of an exact integration over the saddle-point manifold [53]. In this paper we will not perform this task for the two-point function but instead focus on the one-point function. Because of the $U_{A}(1)$ symmetry, the microscopic spectral density already shows a nontrivial structure for eigenvalues close to zero as has been observed in lattice QCD simulations [7, 8]. The discussion of the two-point function will appear in a future publication 54.

The valence quark mass dependence of the chiral condensate was calculated in partially quenched chiral perturbation theory by Golterman and Leung [55] by means of a supersymmetric method introduced by Bernard and Golterman [44, 45]. We use this result to calculate the spectral density in the perturbative domain. Among others we reproduce the slope of the spectral density first derived by Smilga and Stern [56]. When, for virtualities below the Thouless energy, the zero modes are treated exactly, we reproduce the random matrix result for the microscopic spectral density. In fact, we show that 
the partially quenched supersymmetric partition function can be written as an ordinary effective finite volume partition function with two more flavors which are the equivalent of one valence quark flavor and its supersymmetric partner. In this way we obtain a natural explanation of a result first found by Damgaard [57].

The organization of this paper is as follows. Partially quenched chiral perturbation theory is introduced in section 2 . In section 3 we discuss its different domains of validity. The choice of the superunitary measure is motivated in section 4 . In sections 5 and 6 the partially quenched partition function is studied in the domain where it is dominated by the zero modes. The valence quark mass dependence in the quenched limit is calculated in section 5 , and, in section 6 , we show that the microscopic spectral density that follows from the valence quark mass dependence of the chiral condensate coincides with the result from chRMT. In section 7 we calculate the valence quark mass dependence of the condensate by means on pqChPT. The transition from chRMT to pqChPT is discussed in section 8. In section 9 we evaluate the spectral density in the diffusive domain. Concluding remarks are made in section 10. An explicit calculation of the spectral density for $N_{f}=1$ is presented in Appendix A and a short derivation of a Berezinian that occurs in the diagonalization of superunitary transformations is given in Appendix B.

\section{Partially Quenched Chiral Perturbation Theory}

Partially Quenched Chiral Perturbation Theory (pqChPT) is an effective theory describing partially quenched QCD at low energies [45, 59]. This is a theory with both sea quarks, appearing in the fermion determinant, and valence quarks which only appear in the operators. Partially quenched chiral perturbation theory interpolates between standard (unquenched) Chiral Perturbation Theory (ChPT) 58 and completely quenched Chiral Perturbation Theory (qChPT) 60, 61]. In this section we give a brief overview of partially quenched chiral perturbation theory in the formulation of Bernard and Golterman. For more details we refer to the original literature [45].

We consider a QCD-like theory with one valence quark and its supersymmetric partner, both with mass $m_{v}$, and $N_{f}$ unquenched quarks (sea quarks) with mass $m_{s}$. Then the fermion determinant corresponding to the valence quark is cancelled by the contribution from a bosonic ghost quark with the same mass [44]. The full chiral symmetry of the theory is thus the direct product of the supergroups (also called graded groups)

$$
G=U_{L}\left(N_{f}+1 \mid 1\right) \otimes U_{R}\left(N_{f}+1 \mid 1\right)
$$

where one of the $U(1)$ groups is broken by the anomaly. The partially quenched QCD Lagrangian at a given order in the momentum can be approximated by an effective La- 
grangian for the field $U \in S U\left(N_{f}+1 \mid 1\right) \oslash U(1)$ which transforms linearly under the chiral group. (Here, $\oslash$ denotes a semi-direct product.) In terms of the Goldstone fields $U$ is parametrized as

$$
U=\exp (i \sqrt{2} \Phi / F)
$$

where $\Phi$ is a $\left(N_{f}+2\right) \times\left(N_{f}+2\right)$ super-Hermitian matrix field:

$$
\Phi=\left(\begin{array}{cc}
\phi & \chi_{\tilde{\phi}}^{\dagger} \\
\chi & \phi
\end{array}\right)
$$

and $F$ is the pion decay constant. Here, $\phi$ is the $\left(N_{f}+1\right) \times\left(N_{f}+1\right)$ matrix containing the ordinary mesons made of quarks and antiquarks, $\tilde{\phi}$ is the meson with two ghost quarks, and $\chi$, which is a vector of length $N_{f}+1$, represents the fermionic mesons consisting of a ghost-quark and an ordinary anti-quark. We choose a diagonal mass matrix with degenerate sea quark masses resulting in the $\left(N_{f}+2\right) \times\left(N_{f}+2\right)$ quark-mass matrix

$$
\hat{\mathcal{M}}=\operatorname{diag}(m_{v}, \underbrace{m_{s}, \ldots, m_{s}}_{N_{f}}, m_{v}) \text {. }
$$

The corresponding pion masses will be denoted by

$$
M_{i j}^{2}=\left(m_{i}+m_{j}\right) \Sigma_{0} / F^{2} \quad \text { for } \quad i, j=s, v
$$

Expanding the Euclidean effective Lagrangian in terms of the momenta and the quark masses we obtain to lowest order [45, 55]

$$
\mathcal{L}_{\text {eff }}=\frac{F^{2}}{4} \operatorname{Str}\left(\partial_{\mu} U \partial_{\mu} U^{\dagger}\right)-\frac{\Sigma_{0}}{2} \operatorname{Str}\left(\hat{\mathcal{M}} U+\hat{\mathcal{M}} U^{\dagger}\right)+\frac{m_{0}^{2}}{6} \Phi_{0}^{2}+\frac{\alpha}{6} \partial_{\mu} \Phi_{0} \partial_{\mu} \Phi_{0}
$$

where we have added a mass term and a kinetic term for the super- $\eta^{\prime}$ flavor-singlet field $\Phi_{0}=\operatorname{Str}(\Phi)$ with parameters $m_{0}^{2}$ and $\alpha$ (for the definition of the supertrace, Str, (also called graded trace) we refer to [62]). In the next section we will show that the term containing $\alpha$ is sub-leading in the chiral expansion. The quark condensate in the chiral limit is denoted by $\Sigma_{0}$. The partition function corresponding to (23) is defined by

$$
Z_{p q}=\int d U e^{-\int d^{4} x \mathcal{L}_{\mathrm{eff}}}
$$

and will be studied for different ranges of the quark masses.

A physical interpretation of the zero momentum component of the flavor singlet mass term can be obtained by linearizing the square according to

$$
e^{-V \frac{m_{0}^{2}}{6} \Phi_{0}^{2}}=\frac{1}{\sqrt{2 \pi\left\langle\nu^{2}\right\rangle}} \int d \nu \operatorname{Sdet}^{\nu}(U) e^{-\frac{\nu^{2}}{2\left\langle\nu^{2}\right\rangle}},
$$


where

$$
\left\langle\nu^{2}\right\rangle=\frac{F^{2} m_{0}^{2} V}{6}
$$

is the familiar topological susceptibility. For the definition of the superdeterminant, Sdet, (also called graded determinant) we refer to [62]. This result allows us to interpret $m_{0}^{2}$ as a topological contribution to the singlet mass. The partition function with zero total topological charge is obtained by multiplying the Goldstone fields with the $U_{A}(1)$ phase and extending the integration manifold to include this contribution (see 477). In the zero momentum sector, the partition function for $\nu=0$ is thus given by (24) and the Lagrangian (23) without the topological mass term $m_{0}^{2} \Phi_{0}^{2} / 6$ but with the integration domain extended to $U\left(N_{f}+1 \mid 1\right)$. This partition function will be discussed in sections 5 and 6 . Notice that the microscopic spectral density depends on the global topological charge. On the other hand, the spectral density at a macroscopic distance from zero is not sensitive to the global topological charge.

\section{Domains of Validity}

For standard chiral perturbation theory the different domains of its validity were discussed in detail by Gasser and Leutwyler [46]. Since the discussion for partially quenched chiral perturbation theory is quite analogous we refer to this paper for additional arguments.

With the basic scale given by $\epsilon \equiv 1 / L \ll \Lambda_{Q C D}$, we consider five different mass scales: the smallest eigenvalue of the Dirac operator $\lambda_{\min } \sim \epsilon^{4}$, the valence quark mass $m_{v} \sim \epsilon^{\alpha}$, the sea quark mass $m_{s} \sim \epsilon^{\beta}$, the pion momenta $p_{\mu} \sim 1 / L \sim \epsilon$ and the $\eta^{\prime}$ mass $m_{\eta^{\prime}} \sim \Lambda_{\mathrm{QCD}} \sim 1$.

The integral over $U$ in (24) can be split into an integral over the zero momentum modes, $U_{0}$, and the nonzero momentum modes, $\xi(x)$, i.e.

$$
U=U_{0} e^{i \xi(x)}
$$

The two different types of modes are mixed by the mass terms and by the singlet in-

teraction. Keeping only terms up to second order in $\xi$, our Lagrangian can be written as

$$
\begin{aligned}
\int d^{4} x \mathcal{L}_{\mathrm{eff}}= & -\frac{\Sigma_{0}}{2} \operatorname{Str}\left[\hat{\mathcal{M}}\left(U_{0}+U_{0}^{\dagger}\right)\left(V-\frac{1}{2} \int d^{4} x \xi^{2}(x)\right)\right] \\
& +\int d^{4} x\left(\frac{F^{2}}{4} \partial_{\mu} \xi_{a} \partial_{\mu} \xi_{a}+\frac{m_{0}^{2}}{6} \Phi_{0}^{2}+\frac{\alpha}{6} \partial_{\mu} \Phi_{0} \partial_{\mu} \Phi_{0}\right) .
\end{aligned}
$$


The nonzero mode fields are $O(\epsilon)$ whereas the zero mode fields are $O\left(\epsilon^{(4-\alpha) / 2}\right)$. The term that mixes both types of modes is of order $\epsilon^{2}$ independent of the value of $\alpha$ (for $1 \leq \alpha<4$ ). This implies that the mixing term is always subleading. Since the fluctuations of the singlet field are of $O\left(\epsilon^{2}\right)$, the kinetic term of the singlet field is subleading as well.

Since the mass term of the nonzero modes is of order $\epsilon^{\alpha-2}$ the integral over the nonzero modes does not contribute to the mass dependence of the partition function for $\alpha>2$. Therefore, for $\alpha<2$ ( or $m<1 / \Lambda_{\mathrm{QCD}} L^{2}$ ) the mass dependence is completely determined by the zero modes. The crossover-point can be determined more accurately by considering the mass dependence of the propagator of the nonzero modes. We will return to this point in section 8 .

In the chiral limit, $m_{s} \rightarrow 0$, the integrals over the zero momentum modes become perturbative for $m_{v} \gg \lambda_{\text {min }}$, whereas for $m_{v} \ll 1 / \Lambda_{\mathrm{QCD}} L^{2}$ the mass dependence of the partition function is completely determined by the zero momentum modes. In the domain

$$
\lambda_{\min } \ll m_{v} \ll 1 / \Lambda_{\mathrm{QCD}} L^{2}
$$

the valence quark mass dependence of the chiral condensate can thus be determined both from the integral over the zero momentum modes and from a perturbative calculation. In section 8 it will be shown that this consistency condition is satisfied.

Actually, because of the mixing by the singlet mass term, the above discussion has to be refined by considering modes that are eigenvectors of the mass matrix. This is essential for the quenched limit. In this case the relevant mesonic mass matrix in a quark basis is given by 60

$$
\left(\begin{array}{cc}
\frac{m_{0}^{2}}{3}+M_{v v}^{2} & -\frac{m_{0}^{2}}{3} \\
-\frac{m_{0}^{2}}{3} & \frac{m_{0}^{2}}{3}-M_{v v}^{2}
\end{array}\right)
$$

To lowest order in $M_{v v}$ the eigenvalues of this matrix are given by $2 m_{0}^{2} / 3+M_{v v}^{4} /\left(2 m_{0}^{2} / 3\right)$ and $-M_{v v}^{4} /\left(2 m_{0}^{2} / 3\right)$. The zero momentum mode corresponding to the second eigenvalue only becomes perturbative for $m_{v} \gg \epsilon^{2}$, where also the nonzero modes contribute to the mass dependence. We conclude that in the quenched case there is no common domain of validity.

Let us contrast this with the mesonic mass matrix for $N_{f}=1$ which is given by

$$
\left(\begin{array}{ccc}
\frac{m_{0}^{2}}{3}+M_{v v}^{2} & \frac{m_{0}^{2}}{3} & -\frac{m_{0}^{2}}{3} \\
\frac{m_{0}^{2}}{3} & \frac{m_{0}^{2}}{3}+M_{s s}^{2} & -\frac{m_{0}^{2}}{3} \\
-\frac{m_{0}^{2}}{3} & -\frac{m_{0}^{2}}{3} & \frac{m_{0}^{2}}{3}-M_{v v}^{2}
\end{array}\right)
$$

In the chiral limit $\left(M_{s s} \rightarrow 0\right)$ the eigenvalues up to order $M_{v v}^{4}$ are given by $\lambda_{1}=m_{0}^{2}+$ $2 M_{v v}^{4} / 3, \lambda_{2}=-M_{v v}^{2} / \sqrt{3}-M_{v v}^{4} / 3$ and $\lambda_{3}=M_{v v}^{2} / \sqrt{3}-M_{v v}^{4} / 3$. In this case, the zero momentum modes corresponding to the smallest eigenvalue become perturbative for $m_{v} \gg$ 
$\lambda_{\min }$ and a common domain of validity can be identified. For $N_{f}$ massless sea quark flavors, $N_{f}-1$ eigenvalues are zero in the diagonal basis. However, mesons with two sea quarks do not enter in the one-loop calculation of the valence quark mass dependence of the chiral condensate. The smallest non-zero eigenvalue in the valence sector is again of order $M_{v v}^{2}$. Therefore, as for one flavor, a common domain of validity can be found. Of course, if also the sea quark masses are in the range (29) a common domain of validity for the dependence of the chiral condensate on the sea quark masses can be found as well.

\section{Choice of Measure}

For the group integration in (24), we have two different possible choices for the superunitary integration measure: a compact one which was used in 60, 45, and a noncompact one which was introduced in [53, 63, 17] in the context of chiral Random Matrix Theory. It was argued in 17 that a noncompact measure reproduces the invariance of the bosonic degrees of freedom under noncompact $U_{A}(1)$ transformations and therefore should be the appropriate measure for the quenched theory. We stress that for perturbative calculations, as in 60, 45], both measures lead to the correct result.

For the purely quenched theory a decomposition of the compact measure in terms of the eigenvalues of the superunitary matrix $U$ can be written down easily. The compact measure for the group $U(1 \mid 1)$ can thus be written as

$$
D U \sim \frac{d \theta d \phi}{\left|e^{i \theta}-e^{i \phi}\right|^{2}} D U^{\prime},
$$

in the parametrization

$$
U=U^{\prime} \Lambda U^{\prime-1}
$$

with $\Lambda$ a diagonal matrix with eigenvalues $\exp (i \theta)$ and $\exp (i \phi)$. This decomposition can be easily generalized to an arbitrary superunitary group. The generalization of the Berezinian appearing in (32) is given in Appendix B.

The measure for the noncompact version of $U(1 \mid 1)$ is obtained by analytic continuation to imaginary angle $\theta \rightarrow-i s$ with $s \in(-\infty ; \infty)$. This results in the measure

$$
D U \sim \frac{d s d \phi}{\left(e^{s}-e^{i \phi}\right)\left(e^{-s}-e^{-i \phi}\right)} D U^{\prime}
$$

which coincides with the measure that was obtained in [53, 63] after a lengthy calculation. We will show in section 5 that the quenched partition function with this noncompact measure reproduces the quenched valence quark mass dependence of chiral random matrix theory. 
The generalization of this measure to an arbitrary superunitary group appears to be more involved than this naive analytic continuation. However, it can be shown that the integration contours contributing to the discontinuity of the valence quark mass dependence of the chiral condensate across the imaginary axis can be deformed such that only an integral over a compact domain remains [53, 63, 17]. In terms of the partition function this corresponds to replacing the noncompact measure by a compact one. This will be the starting point of the calculation of the microscopic spectral density discussed in section 6. The calculation of the valence quark mass dependence of the chiral condensate for arbitrary $N_{f}$ using a noncompact measure will be discussed elsewhere [64].

\section{Valence Quark Mass Dependence of the Chiral Condensate in the quenched limit}

In this section we will show that in the quenched case the noncompact measure (34) leads to the correct result for the valence quark mass dependence of the chiral condensate. As argued at the end of section 2, the partially quenched partition function in the sector of zero topological charge is obtained by extending the integration over $U$ in (24) to $U\left(N_{f}+1 \mid 1\right)$ and ignoring the singlet channel mass term in (23). In the quenched limit the integration is thus over $U(1 \mid 1)$. With the measure (34) the sector of the zero momentum modes of the partition function (24) in the quenched limit can be written as

$Z(J)=\int \frac{d U d \theta d s}{\left(e^{s}-e^{i \theta}\right)\left(e^{-s}-e^{-i \theta}\right)} \exp \left[\frac{\Sigma_{0} V}{2} \operatorname{Str}\left(\begin{array}{cc}m+J & 0 \\ 0 & m-J\end{array}\right) U\left(\begin{array}{cc}\cos \theta & 0 \\ 0 & \cosh s\end{array}\right) U^{-1}\right]$.

The normalization of this partition function, $Z(0)$, follows from Wegner's theorem for superinvariant functions [69. The integral over $U$ can be performed by means of the supersymmetric version of the Itzykson-Zuber integral [65, 66, 67]. After differentiation with respect to $J$ the result for the valence quark mass dependence of the chiral condensate is

$$
\Sigma(x)=\frac{\Sigma_{0}}{\pi} \int d \theta d s \frac{\cos \theta-\cosh s}{\left(e^{s}-e^{i \theta}\right)\left(e^{-s}-e^{-i \theta}\right)} e^{x(\cos \theta-\cosh s)},
$$

where we have introduced the notation $x=m V \Sigma_{0}$. This integral can be evaluated most conveniently after differentiation with respect to $x$

$$
\partial_{x} \Sigma(x)=\frac{\Sigma_{0}}{4 \pi} \int d \theta d s\left(1-e^{-i \theta-s}\right)\left(1-e^{i \theta+s}\right) e^{x(\cos \theta-\cosh s)}
$$


where the integrand has been simplified by means of the identity

$$
\frac{\cos \theta-\cosh s}{e^{i \theta}-e^{s}}=\frac{1}{2}\left(1-e^{-s} e^{-i \theta}\right) .
$$

The integrals appearing in (37) factorize and can be expressed in terms of the modified Bessel functions $K_{0}$ and $I_{0}$,

$$
\partial_{x} \Sigma(x)=\Sigma_{0}\left[I_{0}(x) K_{0}(x)-I_{1}(x) K_{1}(x)\right] .
$$

The r.h.s. of this equation can be rewritten as $\partial_{x}\left[x I_{0}(x) K_{0}(x)+x I_{1}(x) K_{1}(x)\right]$. This equation can be integrated trivially with an integration constant that follows from the asymptotic limit $\Sigma(x) \rightarrow \Sigma_{0}$ for $x \rightarrow \infty$. The final result is

$$
\Sigma(x)=\Sigma_{0} x\left[I_{0}(x) K_{0}(x)+I_{1}(x) K_{1}(x)\right],
$$

which agrees with the valence quark mass dependence obtained from chiral Random Matrix Theory [51]. In its domain of validity, this result reproduces the valence quark mass dependence found in lattice QCD [51, 50].

\section{Microscopic Spectral Density in the Ergodic Domain}

In this section we consider the zero momentum component of the partially quenched partition function for zero total topological charge. In [53, 17] it was shown that the noncompact integral contributing to the imaginary part of the chRMT partition function can be rewritten as an integral over $[0,2 \pi]$. The spectral density can then be derived from a compactified partition function. By a direct translation of these arguments to the partition function of pqChPT, we obtain for the spectral density

$$
\langle\rho(\lambda)\rangle=\left.\frac{1}{2 \pi} \operatorname{Im} \frac{1}{Z_{p q}^{0}(J=0)} \frac{\partial Z_{p q}^{0}}{\partial J}\right|_{m_{v}=i \lambda, J=0},
$$

where $Z_{p q}^{0}(J)$ is the zero momentum pqChPT partition function with the compact measure defined by

$$
Z_{p q}^{0}(M)=\int d U e^{\frac{\Sigma_{0}}{2} \operatorname{Str}\left(M U+M U^{\dagger}\right)}
$$

Here, the integral is over $U\left(N_{f}+1 \mid 1\right)$ corresponding to the sector of zero topological charge. The mass-matrix $M$ is diagonal with matrix elements $\left(m_{v}+J, m_{s}, \cdots, m_{s}, m_{v}-J\right)$.

In a diagonal representation of the of superunitary matrices $U, Z_{p q}^{0}$ for $N_{f}$ flavors can be written as

$$
Z_{p q}^{0}(M)=\int \prod d \theta_{k} d U|B(\Lambda)|^{2} e^{\frac{\Sigma_{0} V}{2} \operatorname{Str} M U\left(\Lambda+\Lambda^{*}\right) U^{-1}}
$$


Here, $|B(\Lambda)|^{2}$ is the Berezinian for the transformation to eigenvalues and eigenvectors as new integration variables (see Appendix B). The quantity $B(\Lambda)$ is given by

$$
B(\Lambda)=\frac{\prod_{k<l}\left(\Lambda_{k}^{b}-\Lambda_{l}^{b}\right) \prod_{k<l}\left(\Lambda_{k}^{f}-\Lambda_{l}^{f}\right)}{\prod_{k, l}\left(\Lambda_{k}^{b}-\Lambda_{l}^{f}\right)},
$$

where the bosonic and fermionic eigenvalues of $U$ are denoted by $\Lambda_{k}^{b}$ and $\Lambda_{k}^{f}$, respectively. In our case we have $N_{f}+1$ fermionic eigenvalues and one bosonic eigenvalue. In the quenched case we use the supersymmetric normalization with $Z_{p q}^{0}(J=0)=1$ whereas in the partially quenched case $Z_{p q}^{0}(J=0)=Z_{N_{f}}^{\text {eff }}$ (defined in eq. (10)). Here, and below the constants multiplying the partition function are absorbed in the integration measure. The integration over $U \in U\left(N_{f}+1 \mid 1\right)$ can be performed by means of a supersymmetric version of the Itzykson-Zuber integral 665, 66, 67]. For $J \neq 0$ this results in

$$
Z_{p q}^{0}(M)=\int \prod_{k} d \theta_{k} \frac{|B(\Lambda)|^{2}}{B\left(\cos \theta_{k}\right) B\left(M_{k} V \Sigma_{0}\right)} e^{-M_{N_{f}+1} V \Sigma_{0} \cos \theta_{N_{f}+1}} \operatorname{det}_{0 \leq k, l \leq N_{f}} e^{M_{k} V \Sigma_{0} \cos \theta_{l}} .
$$

For $J=0$, the Grassmannian components of the integration paths lead to additional contributions. These so called Efetov-Wegner [68, 69] terms only enter in the normalization of the partition function (see [70, 63, 30, 71] for more discussion of this point).

We evaluate this integral in the chiral limit, $m_{s} \rightarrow 0$, for a fixed value of the valence quark mass. In this limit the ratio of the determinant and $B\left(M_{k} V \Sigma_{0}\right)$ is finite. In Appendix A, we evaluate the integral for $N_{f}=1$ and indicate how it can be calculated for arbitrary $N_{f}$. In the remainder of this section we show that this generating function can be expressed as an ordinary effective finite volume partition function (see (10)) with $N_{f}+2$ flavors.

For arbitrary $N_{f}$ the partition function (45) can be written as

$Z_{p q}^{0}(M)=\mathcal{N} \int \prod_{k} d \theta_{k} \prod_{0 \leq k<l \leq N_{f}} \frac{\omega\left(\theta_{k}, \theta_{l}\right)}{\left(\mu_{k}-\mu_{l}\right)} \prod_{0 \leq k \leq N_{f}} \frac{\left(\mu_{k}-\mu_{N_{f}+1}\right)}{\omega\left(\theta_{k}, \theta_{N_{f}+1}\right)} e^{-\mu_{N_{f}+1} \cos \theta_{N_{f}+1}} \operatorname{det}_{0 \leq k, l \leq N_{f}} e^{\mu_{k} \cos \theta_{l}}$,

with $\mu_{0}=\left(m_{v}+J\right) V \Sigma_{0}, \mu_{N_{f}+1}=\left(m_{v}-J\right) V \Sigma_{0}$ and $\mu_{k}=m_{s} V \Sigma_{0}, k=1, \cdots N_{f}$. For equal sea quark masses the ratio of the last factor and $\prod_{k<l}\left(\mu_{k}-\mu_{l}\right)$ has to be understood as a limit. The normalization factor that enters in the partition function is denoted by $\mathcal{N}$. The function $\omega(\theta, \phi)$ defined by

$$
\omega(\theta, \phi)=\frac{\left|e^{i \theta}-e^{i \phi}\right|^{2}}{\cos \theta-\cos \phi}
$$

has the important property that

$$
\omega(\theta, \phi)=\frac{4}{\omega(\theta,-\phi)}=4 \frac{\cos \theta-\cos \phi}{\left|e^{i \theta}-e^{-i \phi}\right|^{2}} .
$$


This allows us to bring all factors $\omega\left(\theta_{k}, \theta_{l}\right)$ into the numerator. Slightly rearranging the different factors and changing the sign of $\mu_{N_{f}+1}$ we can write the partition function in the more symmetric form

$$
Z_{p q}^{0}(\tilde{M})=\mathcal{N} \prod_{0 \leq k \leq N_{f}}\left(\mu_{k}^{2}-\mu_{N_{f}+1}^{2}\right) \int \prod_{k} d \theta_{k} \prod_{0 \leq k<l \leq N_{f}+1} \frac{4 \omega\left(\theta_{k}, \theta_{l}\right)}{\left(\mu_{k}-\mu_{l}\right)} \frac{\operatorname{det}_{0 \leq k, l \leq N_{f}+1} e^{\mu_{k} \cos \theta_{l}}}{N_{f}+2}
$$

where the diagonal mass matrix $\tilde{M}$ is defined by $\tilde{M}=\left(m_{v}+J, m_{s}, \cdots, m_{s},-m_{v}+J\right)$. In this integral we recognize the factors that occur in the ordinary Itzykson-Zuber integral. It can thus be rewritten as

$$
Z_{p q}^{0}(\tilde{M})=\mathcal{N}^{\prime} \prod_{0 \leq k \leq N_{f}}\left(\mu_{k}^{2}-\mu_{N_{f}+1}^{2}\right) \int d U \prod_{k} d \theta_{k}\left|\Delta\left(e^{i \theta_{k}}\right)\right|^{2} e^{\frac{\Sigma_{0} V}{2} \operatorname{Tr} \tilde{M} U \cos \left(\theta_{k}\right) U^{-1}}
$$

where the $U$-integral is over $U\left(N_{f}+2\right)$. Up to a constant factor this is exactly the effective finite volume partition function for $N_{f}+2$ flavors. In the microscopic limit with quark masses of order $\sim 1 / V \Sigma_{0}$, this partition function is equivalent to the random matrix partition function with $N_{f}+2$ flavors. For masses in the microscopic domain we thus have

$$
Z_{p q}^{0}(\tilde{M})=\mathcal{N}^{\prime \prime} \prod_{0 \leq k \leq N_{f}}\left(\mu_{k}^{2}-\mu_{N_{f}+1}^{2}\right) Z_{R M T}^{\left(N_{f}+2\right)}\left(m_{v}+J, m_{1}, \ldots, m_{N_{f}},-m_{v}+J\right)
$$

where $Z_{R M T}^{\left(N_{f}+2\right)}$ is the chRMT partition function as defined in (11) for $N_{f}+2$ flavors, zero topological charge and $\beta=2$. Since the RMT partition function is even in all masses (51) can be rewritten as

$$
Z_{p q}^{0}(\tilde{M})=\mathcal{N}^{\prime \prime} \prod_{0 \leq k \leq N_{f}}\left(\mu_{k}^{2}-\mu_{N_{f}+1}^{2}\right) Z_{R M T}^{\left(N_{f}+2\right)}\left(m_{v}+J, m_{1}, \ldots, m_{N_{f}}, m_{v}-J\right) .
$$

If we notice that $\mu_{0}^{2}-\mu_{N_{f}+1}^{2}=4 m_{v} \Sigma_{0} V J+O\left(J^{2}\right)$, the spectral density is easily obtained from (41) by differentiating with respect to $J$ and setting $m_{v}=i \lambda$. After taking the imaginary part we obtain for the microscopic limit of the spectral density defined in (4),

$$
\rho_{S}(u)=\frac{1}{2}|u| \prod_{1 \leq k \leq N_{f}}\left(\mu_{k}^{2}+u^{2}\right) \frac{Z_{R M T}^{\left(N_{f}+2\right)}\left(i u, \mu_{1}, \cdots, \mu_{N_{f}}, i u\right)}{Z_{R M T}^{N_{f}}\left(\mu_{1}, \cdots, \mu_{N_{f}}\right)},
$$

where $u=\lambda V \Sigma_{0}$. The denominator arises from the normalization $Z_{p q}^{0}(J=0)$ of the partially quenched partition function. The overall normalization is such that $\rho(\lambda) \rightarrow \Sigma_{0} / \pi$ for $\lambda \rightarrow \infty$. This result coincides with the expression first derived by Damgaard [57]. Our derivation provides a natural explanation of their result. The two extra flavors are a valence quark and its supersymmetric partner. Remarkably, in the context of chRMT, 
the spectral density for $\lambda$ not close to zero can still be expressed as the ratio of the same two partition functions. It was shown in [57] that (53) can be reduced to the analytical expression for the microscopic spectral density of chRMT which, in the case of zero quark masses, is given by [72, 48]

$$
\rho_{S}(u)=\frac{u}{2}\left[J_{N_{f}}^{2}(u)-J_{N_{f}+1}(u) J_{N_{f}-1}(u)\right] .
$$

We have thus shown that the microscopic spectral density of chRMT can be derived from the partition function of partially quenched chiral perturbation theory. The valence quark mass dependence of the chiral condensate can be obtained from (54) by replacing the sum in (13) by an integral. In terms of the microscopic variables, $u=\lambda V \Sigma_{0}$ and $x=m V \Sigma_{0}$, this amounts to

$$
\Sigma(x)=\Sigma_{0} \int_{0}^{\infty} d u \frac{1}{V \Sigma_{0}}\left\langle\rho\left(\frac{u}{V \Sigma_{0}}\right)\right\rangle \frac{2 x}{u^{2}+x^{2}} .
$$

In the thermodynamic limit the spectral density in this equation can be replaced by the microscopic spectral density (54) resulting in

$$
\frac{\Sigma(x)}{\Sigma_{0}}=x\left[I_{N_{f}}(x) K_{N_{f}}(x)+I_{N_{f}+1}(x) K_{N_{f}-1}(x)\right] .
$$

By using the duality between flavor and topology [49] the result for the sector of topological charge $\nu$ is obtained by replacing $N_{f} \rightarrow N_{f}+|\nu|$.

The calculation of the valence quark mass dependence of the chiral condensate from the partition function with a noncompact measure would justify both the choice of the noncompact measure and the generalization of the arguments from [53, 18] relating the compact and the noncompact measures. In a future publication 64 we will come back to this point. In the next section we perform a one-loop calculation of the valence quark mass dependence of the chiral condensate. In section 8 it is shown that the zero momentum component of this result coincides with the asymptotic expansion of (56) up to this order.

\section{Valence Quark Mass Dependence of the Chiral Condensate in Partially Quenched Chiral Perturbation Theory}

From a computational point of view, qChPT and pqChPT are not very different. For example, it is much easier to use the quark basis [45, 59, 60, 61] with mesons $\bar{q}_{i} q_{j}$ and corresponding masses $M_{i j}^{2}=\Sigma_{0}\left(m_{i}+m_{j}\right) / F^{2}$ (to lowest order), $i, j=s, v$. To lowest order, the bilinear quadratic form in the meson fields is diagonal in the off-diagonal mesons fields with quark content $\bar{q}_{i} q_{j}$ (with $i \neq j$ ). It is therefore trivial to write down the propagator for these mesons. However, there are non-trivial interactions between the 
diagonal mesons. Nevertheless it is still possible, as shown by Bernard and Golterman [45], to invert the inverse propagator analytically. The result for one valence quark mass is 45 :

$$
G_{i j}(p)=\frac{\delta_{i j} \epsilon_{i}}{p^{2}+M_{i i}^{2}}-\frac{m_{0}^{2}+\alpha p^{2}}{\left(3+N_{f} \alpha\right)} \frac{\left(p^{2}+M_{s s}^{2}\right)}{\left(p^{2}+M_{i i}^{2}\right)\left(p^{2}+M_{j j}^{2}\right)\left(p^{2}+M_{\eta^{\prime}}^{2}\right)},
$$

where $\epsilon_{i}$ is 1 for the usual mesons and -1 otherwise. The mass of the super- $\eta^{\prime}$ is given by:

$$
M_{\eta^{\prime}}^{2}=\frac{M_{s s}^{2}+N_{f} m_{0}^{2} / 3}{1+N_{f} \alpha / 3}=M_{s s}^{2} \frac{1+N_{f} y}{1+N_{f} \alpha / 3},
$$

where $y=m_{0}^{2} / 3 M_{s s}^{2}$ [55].

The quenched limit is obtained in the limit of infinite sea quark masses at fixed values of the valence quark mass and $m_{0}$. On the other hand, standard ChPT can be recovered in two ways. First, in the sector of the mesons with two sea quarks, in the limit where the sea quark masses are much less than the mass of the super- $\eta^{\prime}$ particle. In that limit the super- $\eta^{\prime}$ can be integrated out of the effective partition and standard chiral perturbation theory with $N_{f}$ flavors is recovered. The propagator of the mesons corresponding to the sea quark masses is given by

$$
G_{s s}(p)=\frac{1}{p^{2}+M_{s s}^{2}}-\frac{1}{N_{f}} \frac{1}{p^{2}+M_{s s}^{2}},
$$

which is just the standard propagator in a quark basis with the singlet channel projected out. Second, we consider the limit in which the valence quark masses and the sea quark masses are equal and both are much less than the super- $\eta^{\prime}$ mass. Then, the super- $\eta^{\prime}$ mass decouples from the theory and, to one-loop order, the contribution from mesons containing valence quarks is cancelled by the superpartners. We again recover chiral perturbation theory for $N_{f}$ flavors in both the valence quark sector and the sea quark sector.

In this work, we are mainly interested in the nonanalytic terms in the quark masses contributing to the chiral condensate at one-loop level. We will ignore the corrections coming from the $O\left(p^{4}\right)$ effective Lagrangian and its unknown effective coupling constants. We will consider the limit where the valence quark mass and the sea quark masses are much less than the $\eta^{\prime}$ mass. Therefore, contributions from $\Phi_{0}$ loops proportional to $\log M_{\eta^{\prime}}^{2}$ will be ignored as well [55].

The thermodynamic limit of the valence quark mass dependence was already calculated by Golterman and Leung [55]. Here, we consider the generalization of their result to finite volume. The volume dependence of the chiral condensate in standard chiral perturbation theory has already received a great deal of attention in the literature 58, 73. We refer to these works for technical details. In order to obtain the volume dependence of the quark 
condensate to one-loop, essentially one has to replace the infinite volume pion propagator by

$$
\Delta\left(M^{2}\right)=\frac{1}{V} \sum_{p} \frac{1}{p^{2}+M^{2}}
$$

where the sum is over the momenta in the box. In the thermodynamic limit the sum can be replaced by an integral resulting in (in four Euclidean dimensions)

$$
\Delta\left(M^{2}\right)=\frac{1}{16 \pi^{2}} M^{2} \ln \frac{M^{2}}{\Lambda^{2}}
$$

where $\Lambda$ is the cutoff.

The generalization of the one-loop result for the valence quark mass dependence of the chiral condensate (denoted by a subscript $v$ ) to finite volume is then given by

$$
\begin{aligned}
\Sigma_{v}=\Sigma_{0}\left[1-\frac{1}{F^{2}}\right. & \left\{N_{f} \Delta\left(M_{s v}^{2}\right)-\frac{\alpha+3 y^{2} N_{f}}{3\left(1+N_{f} y\right)^{2}} \Delta\left(M_{v v}^{2}\right)\right. \\
& \left.\left.+\left(M_{s s}^{2} \frac{y}{1+N_{f} y}-M_{v v}^{2} \frac{\alpha+3 N_{f} y^{2}}{3\left(1+N_{f} y\right)^{2}}\right) \partial_{M_{v v}^{2}} \Delta\left(M_{v v}^{2}\right)\right\}\right] .
\end{aligned}
$$

Notice that $\partial_{M^{2}} \Delta\left(M^{2}\right)=-\frac{1}{V} \sum_{p} 1 /\left(p^{2}+M^{2}\right)^{2}$. We study the valence quark mass dependence of $\Sigma_{v}$ for several special cases. The first limit of interest is the case that both the valence quark mass and the sea quark masses are much less than the $\eta^{\prime}$ mass. This corresponds to taking the limit $y \rightarrow \infty$ of the expression obtained in (62)

$$
\Sigma_{v}=\Sigma_{0}\left[1-\frac{1}{N_{f} F^{2}}\left\{N_{f}^{2} \Delta\left(M_{s v}^{2}\right)-\Delta\left(M_{v v}^{2}\right)+\left(M_{s s}^{2}-M_{v v}^{2}\right) \partial_{M_{v v}^{2}} \Delta\left(M_{v v}^{2}\right)\right\}\right] .
$$

In the thermodynamic limit this reduces to

$$
\Sigma_{v} \simeq \Sigma_{0}\left[1-\frac{\Sigma_{0}}{16 \pi^{2} F^{4} N_{f}}\left\{N_{f}^{2}\left(m_{v}+m_{s}\right) \log \frac{m_{v}+m_{s}}{2 \mu}+2\left(m_{s}-2 m_{v}\right) \log \frac{m_{v}}{\mu}\right\}\right],
$$

where $\mu=\Lambda^{2} F^{2} / 2 \Sigma_{0}$. Notice that the valence quark mass occurs in the operator. Three special cases of this result are to be mentioned. For $m_{s}=m_{v}$ we recover the one-loop result for standard chiral perturbation theory.

For valence quark masses in the range $m_{s} \ll m_{v} \ll m_{0}$ the valence quark mass dependence of $\Sigma_{v}$ is given by

$$
\Sigma_{v} \simeq \Sigma_{0}\left[1-\frac{\Sigma_{0}}{16 \pi^{2} F^{4}} \frac{N_{f}^{2}-4}{N_{f}} m_{v} \log \frac{m_{v}}{\mu}\right] .
$$

Below we will show that this result leads to the expression for the slope of the spectral density derived in 56. 
For valence quark masses in the range $m_{v} \ll m_{s} \ll m_{0}$ the expression (64) reduces to

$$
\Sigma_{v} \simeq \Sigma_{0}\left[1-\frac{\Sigma_{0}}{16 \pi^{2} F^{4} N_{f}}\left\{2 m_{s} \log \frac{m_{v}}{\mu}\right\}\right] .
$$

The expectation is that on this scale for the valence quark mass the result for $\Sigma_{v}$ should reduce to the quenched result.

Let us consider the quenched limit of (62). This limit amounts to taking the sea quark mesons much heavier than the $\eta^{\prime}$ mass, i.e. the limit $y \rightarrow 0$ while $M_{s s}^{2} y=m_{0}^{2} / 3$. In this limit (62) reduces to

$$
\begin{aligned}
\Sigma_{v}^{Q} & =\Sigma_{0}\left[1-\frac{1}{3 F^{2}}\left\{-\alpha \Delta\left(M_{v v}^{2}\right)+\left(m_{0}^{2}-\alpha M_{v v}^{2}\right) \partial_{M_{v v}^{2}} \Delta\left(M_{v v}^{2}\right)\right\}\right] \\
& \simeq \Sigma_{0}\left[1-\frac{1}{48 \pi^{2} F^{2}}\left(m_{0}^{2}-2 \alpha M_{v v}^{2}\right) \log \frac{M_{v v}^{2}}{\Lambda^{2}}\right] \text { for } \quad V \rightarrow \infty
\end{aligned}
$$

where, in the second line, we have only quoted the infrared singular terms. For a valence quark masses much less than $m_{0}$, the term proportional to $\alpha$ can be ignored.

Let us return to (66). We observe that it corresponds to the quenched result provided that $m_{0}^{2}=3 M_{s s}^{2} / N_{f}$. Indeed, for the low-momentum modes we expect that, according to the Witten-Veneziano formula, the topological contribution to the $\eta^{\prime}$ mass is determined by the global topological susceptibility. As dictated by the chiral Ward identity, this quantity is suppressed by the screening of topological charge due to light sea quarks and is given by 74

$$
\left\langle\nu^{2}\right\rangle=\frac{F^{2} M_{s s}^{2}}{2 N_{f}}
$$

If we combine this result with the relation between $m_{0}$ and $\left\langle\nu^{2}\right\rangle$ given in (26) we recover the previously mentioned condition.

\section{From pqChPT to chRMT}

Let us study the valence quark mass dependence of $\Sigma_{v}$ in the chiral limit of the sea quark masses and valence quark masses in the domain

$$
\lambda_{\min } \ll m_{v} \ll \frac{1}{L^{2} \Lambda_{\mathrm{QCD}}} .
$$

As has been argued in section 3, in this domain both the random matrix expressions (which follow from the zero momentum component of the partition function (24)) and 
the pqChPT results (62) are valid. For $m_{v}$ in the range (70) the propagator $\Delta\left(M_{v v}^{2}\right)$ is dominated by the zero momentum mode resulting in

$$
\Delta\left(M_{v v}^{2}\right) \sim \frac{1}{M_{v v}^{2} V}
$$

The valence quark mass dependence then reduces to

$$
\begin{aligned}
\Sigma_{v} & \sim \Sigma_{0}\left(1-\frac{N_{f}}{V M_{s v}^{2} F^{2}}\right) \\
& \sim \Sigma_{0}\left(1-\frac{N_{f}}{m_{v} V \Sigma_{0}}\right) .
\end{aligned}
$$

In section 6 we derived the valence quark mass dependence of the chiral condensate from the zero momentum pqChPT partition function. The result that was obtained by integrating the microscopic spectral density coincides with the expression found from chRMT [51]. The asymptotic expansion in powers of $1 / x \equiv 1 / m_{v} V \Sigma_{0}$ of $\Sigma_{R M T}(x)$ given in (56) with the replacement $N_{f} \rightarrow N_{f}+|\nu|$ is given by

$$
\frac{\Sigma_{R M T}(x)}{\Sigma_{0}} \sim 1-\frac{N_{f}+|\nu|}{x}+\frac{\left(N_{f}+|\nu|+\frac{1}{2}\right)\left(N_{f}+|\nu|-\frac{1}{2}\right)}{2 x^{2}}+O\left(\frac{1}{x^{3}}\right) .
$$

In the chiral limit the global topological susceptibility is completely screened. For $\nu=0$ we thus find

$$
\Sigma(x) \sim \Sigma_{0}\left(1-\frac{N_{f}}{m_{v} V \Sigma_{0}}\right)
$$

which coincides with the result for chiral perturbation theory in this domain.

In the intermediate region where $M_{v v} L \sim 1$, pqChPT is still meaningful as a perturbation theory. The finite volume effects are embedded in the pion propagator. For the propagator of a free scalar particle in a finite volume we have [46, 73],

$$
\begin{aligned}
\Delta\left(M^{2}\right) & =\frac{1}{16 \pi^{2}} M^{2} \log \frac{M^{2}}{\Lambda^{2}}+g_{1}\left(M^{2}, L\right), \\
\partial_{M^{2}} \Delta\left(M^{2}\right) & =\frac{1}{16 \pi^{2}}\left(1+\log \frac{M^{2}}{\Lambda^{2}}\right)-g_{2}\left(M^{2}, L\right),
\end{aligned}
$$

where

$$
g_{r}\left(M^{2}, L\right)=\frac{1}{16 \pi^{2}} \int_{0}^{\infty} d \lambda \lambda^{r-3} \sum_{\mathbf{n} \neq 0} e^{-\lambda M^{2}-\mathbf{n}^{2} L^{2} / 4 \lambda}
$$

and the sum is over a four dimensional lattice of integers. For $1 / \Lambda_{Q C D} L^{2} \ll M \ll 1 / L$ the functions $g_{r}$ can be expanded in powers of $M$ resulting in

$$
\begin{aligned}
& g_{1}\left(M^{2}, L\right)=\frac{1}{M^{2} L^{4}}-\frac{\beta_{1}}{L^{2}}+\frac{\beta_{2}}{2} M^{2}+O\left(M^{4}\right) \\
& g_{2}\left(M^{2}, L\right)=\frac{1}{M^{4} L^{4}}+\frac{1}{16 \pi^{2}} \log \left(M^{2} L^{2}\right)-\frac{\beta_{2}}{2}+O\left(M^{2}\right)
\end{aligned}
$$


where $\beta_{1}=0.140461$ and $\beta_{2}=-0.020305$ are shape coefficients of a 4 -dimensional cubic box [73. Let us consider the chiral limit $m_{s} \rightarrow 0$. Then, in the intermediate range, the asymptotic result (72) is reproduced. For $M L \sim 1$ the expansion (77) breaks down and the functions $g_{r}$ have to be evaluated numerically.
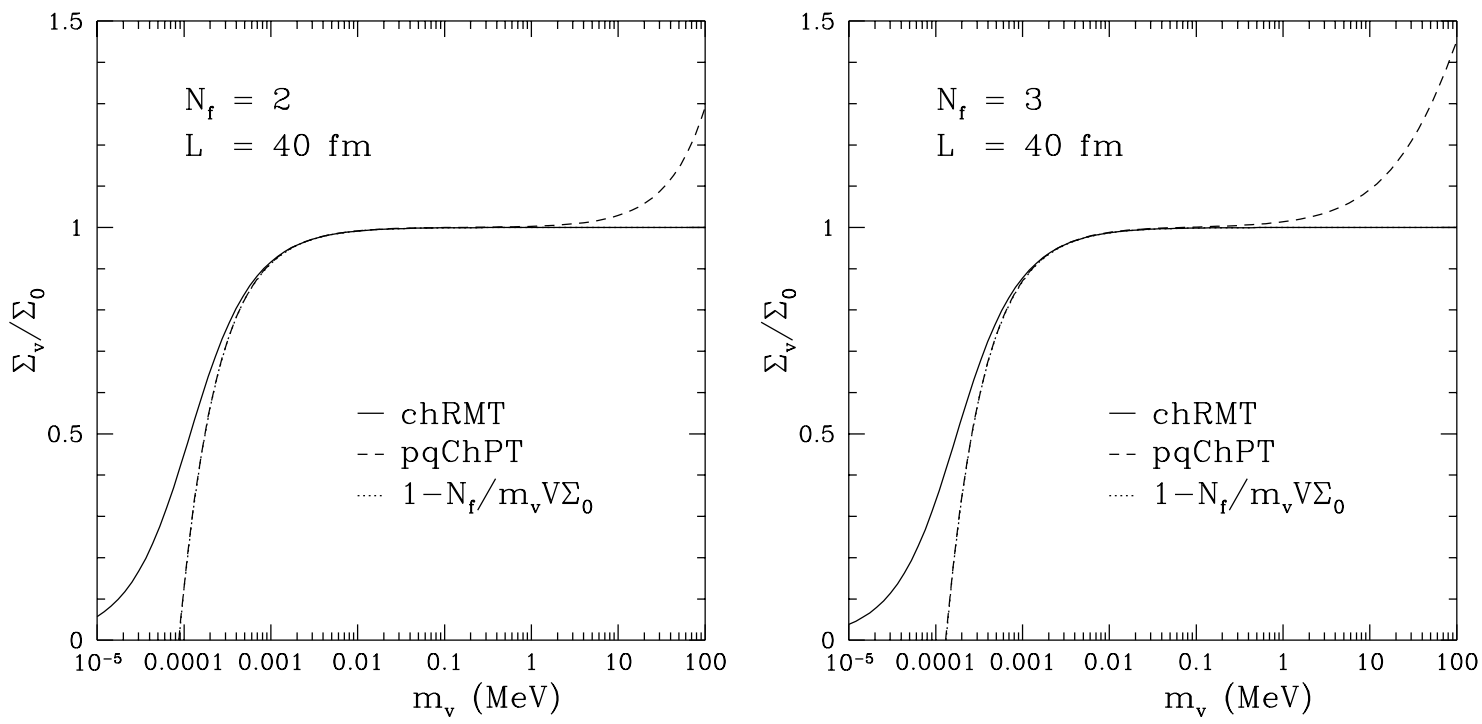

Fig. 1. The valence quark mass dependence of the chiral condensate $\Sigma_{v} / \Sigma_{0}$ as a function of the valence quark mass in $\mathrm{MeV}$ for two (left) and three (right) massless flavors.

In Fig. 1 we show the ratio $\Sigma_{v} / \Sigma_{0}$ as a function of the valence quark mass in $M e V$ for two (left) and three (right) massless flavors for a box of size $(40 \mathrm{fm})^{4}$. The dashed curves represent the general expression for the valence quark mass dependence given in (63) in the limit $m_{s} \rightarrow 0$ and $\Delta\left(M^{2}\right)$ and $\partial_{M^{2}} \Delta\left(M^{2}\right)$ given by (75) with the exact numerical result for the functions $g_{r}$. If the numerical results for the $g_{r}$ are replaced by the leading term of the small mass expansion (77) we obtain the dotted curves (which first lie almost exactly underneath the pqChPT and then underneath the chRMT curves). The random matrix result for the valence quark masses (56) for $N_{f}=2$ or $N_{f}=3$ and $\nu=0$ is given by the solid curves. We stress that this result can also be derived from the pqChPT partition function as shown in section 6 . The results in this figure have been calculated for $\Lambda=770 \mathrm{MeV}, F=93 \mathrm{MeV}$ and $\Sigma_{0} / F^{2}=1660 \mathrm{MeV}$. For these values the Thouless energy is $E_{c} \equiv F^{2} / \Sigma_{0} L^{2} \approx 0.015 \mathrm{MeV}$. We observe a region where the random matrix results and the pqChPT results are in agreement. The size of this region is consistent with the range (29). For $N_{f}=2$ this region appears larger than for $N_{f}=3$ because the leading correction vanishes in the thermodynamic limit for $N_{f}=2$. Because of the large box size, the $1 / L^{2}$ terms are almost invisible in this figure at the point where the nonzero momentum modes become important. 

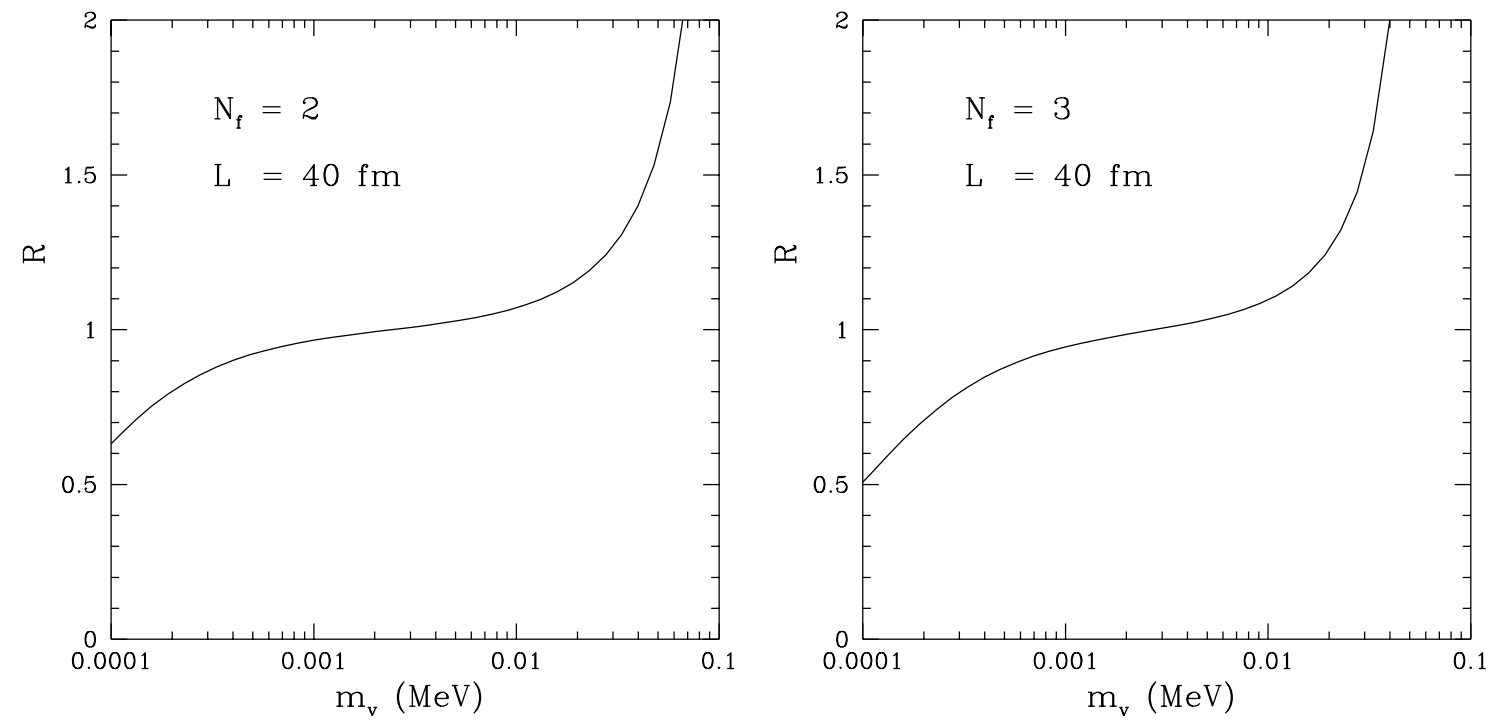

Fig. 2. The ratio $R=\left(\Sigma_{R M T}-\Sigma_{0}\right) /\left(\Sigma_{v}-\Sigma_{0}\right)$ versus the valence quark mass $m_{v}$ for two and three massless flavors.

In order to study the point where the nonzero momentum modes become important we study the ratio

$$
R=\frac{\Sigma_{R M T}-\Sigma_{0}}{\Sigma_{v}-\Sigma_{0}}
$$

According to (72) and (73) this ratio is close to 1 in the range (70). In Fig. 2 we plot this ratio as a function of the valence quark mass $m_{v}$ for a box size of $(40 \mathrm{fm})^{4}$ and $N_{f}=2$ (left) or $N_{f}=3$ (right). At intermediate masses we indeed observe a plateau where chRMT and pqChPT are in agreement. Notice that in the center of the overlap region (70), where $m_{v}$ scales as $1 / L^{3}$, the deviation of this ratio from 1 is of order $1 / L$. Indeed, for larger volumes we have observed a more pronounced plateau. The Thouless energies can be read off from these pictures. For $N_{f}=2$ and $N_{f}=3$, we find values of around 0.01-0.02 $\mathrm{MeV}$ which is in good agreement with the estimate from $E_{c}=F^{2} / \Sigma_{0} L^{2}$.

\section{Spectral Density in the Diffusive Domain}

As already has been remarked in section 1, the spectral density is obtained from the discontinuity of the valence quark mass dependence of the chiral condensate along the imaginary axis. First, notice that the quark mass always occurs in the renormalization group invariant combination $m\langle\bar{q} q\rangle$ with the sign of $\langle\bar{q} q\rangle$ opposite to the sign of $m$. This combination can be rewritten as $|m| \Sigma$. For similar reasons, $\Sigma_{0}$ in the expressions for the valence quark mass dependence of the chiral condensate is actually multiplied by $\left|m_{v}\right| / m_{v}$ 
with $\left|m_{v}\right|=\sqrt{m_{v}^{2}}$. From the elementary identity $\left.\left.\lim _{\epsilon \rightarrow 0}\left[\sqrt{(}-x^{2}+i \epsilon\right)-\sqrt{(}-x^{2}-i \epsilon\right)\right] / i x=2$ we thus conclude that the discontinuity of the first term in (64) is equal to 2. According to (14) this results in a contribution to the spectral density of $\Sigma_{0} / \pi$ which is in agreement with the Banks-Casher formula (1). The discontinuities that enter in the calculation of the spectral density from the one-loop result for the valence quark mass dependence of $\Sigma_{v}$ are given by

$$
\begin{aligned}
& \left.\operatorname{Disc}\right|_{m_{v}=i x}\left(\frac{\left|m_{v}\right|}{m_{v}}\right)=2, \\
& \left.\operatorname{Disc}\right|_{m_{v}=i x}\left(\frac{\left|m_{v}\right|}{m_{v}} \log \left(m_{s}+\left|m_{v}\right|\right)\right)=\log \left(m_{s}^{2}+x^{2}\right), \\
& \left.\operatorname{Disc}\right|_{m_{v}=i x}\left(\log \left(m_{s}+\left|m_{v}\right|\right)\right)=2 i \arctan \frac{x}{m_{s}}, \\
& \left.\operatorname{Disc}\right|_{m_{v}=i x} \log \left|m_{v}\right|=i \pi .
\end{aligned}
$$

The spectral density can be calculated from the one-loop result for the valence quark mass dependence of $\Sigma_{v}$ (64) by means of $\langle\rho(\lambda)\rangle / V=\left.\operatorname{Disc}\right|_{m_{v}=i \lambda} \Sigma_{v}\left(m_{v}\right) / 2 \pi$. The result is

$$
\frac{\langle\rho(\lambda)\rangle_{m_{s}}}{V}=\frac{\Sigma_{0}}{\pi}\left[1+\frac{\Sigma_{0}}{32 \pi^{2} N_{f} F^{4}}\left\{2 N_{f}^{2}|\lambda| \operatorname{Arctg} \frac{|\lambda|}{m_{s}}-4 \pi|\lambda|-N_{f}^{2} m_{s} \log \frac{\lambda^{2}+m_{s}^{2}}{\mu^{2}}-4 m_{s} \log \frac{|\lambda|}{\mu}\right\}\right] .
$$

For $m_{s} \neq 0$ the spectral density diverges logarithmically. In the limit $\lambda \gg m_{s}$ this expression reduces to

$$
\frac{\langle\rho(\lambda)\rangle_{m_{s}}}{V}=\frac{\Sigma_{0}}{\pi}\left[1+\frac{\left(N_{f}^{2}-4\right) \Sigma_{0}}{32 \pi^{2} N_{f} F^{4}}|\lambda|\right]
$$

which was first obtained by Smilga and Stern [56].

Our expression for the spectral density can be used to evaluate the connected contribution to the integrated three-point scalar correlator,

$$
K^{a b c}=\int d^{4} x d^{4} y d^{4} z\left\langle 0\left|S^{a}(x) S^{b}(y) S^{c}(z)\right| 0\right\rangle,
$$

with $S^{a}=\bar{q} t^{a} q$ and $t^{a}$ is one of the generators of the flavor $S U\left(N_{f}\right)$ group in the normalization $\operatorname{Tr}\left(t^{a} t^{b}\right)=\delta^{a b} / 2$. This correlator can be expressed in terms of the spectral density by 56

$$
K^{a b c}=-m_{s} d^{a b c} \int_{0}^{\infty} d \lambda \frac{m_{s}^{2}-3 \lambda^{2}}{\left(m_{s}^{2}+\lambda^{2}\right)^{3}}\langle\rho(\lambda)\rangle_{m_{s}}
$$

where $d^{a b c}$ are the symmetric structure constants of $S U\left(N_{f}\right)$. By substitution of the expression (83) for the spectral density we then obtain

$$
\frac{1}{V} K^{a b c}=d^{a b c} \frac{N_{f}^{2}-12}{N_{f} m_{s}} \frac{\Sigma_{0}^{2}}{128 \pi^{2} F^{4}}
$$


which is in complete agreement with the result obtained by Smilga and Stern using standard ChPT [56]. Notice that both terms of order $O\left(m_{s}^{0}\right)$ and of $O\left(m_{s}^{1}\right)$ in (83) contribute to this result. The existence of contributions of this type was already pointed out in [56], but partially quenched chiral perturbation theory enabled us to actually calculate them.

The spectral density in the quenched case can be obtained in exactly the same way by calculating the discontinuities of the valence quark mass dependence across the imaginary axis. The result is

$$
\frac{\left\langle\rho^{Q}(\lambda)\right\rangle}{V}=\frac{\Sigma_{0}}{\pi}\left[1-\frac{m_{0}^{2}}{48 \pi^{2} F^{2}} \log \frac{|\lambda|}{\mu}-\frac{\Sigma_{0} \alpha}{24 \pi F^{4}}|\lambda|\right] .
$$

A logarithmic divergence of the spectral density in the quenched limit has indeed been observed in quenched instanton liquid simulations [43]. This result allows us to interpret the coefficient of $\log |\lambda|$ in the limit $\lambda \ll m_{s}$ of (83) in terms of the screened topological charge (69).

\section{Conclusions}

We have studied the spectral density of the QCD Dirac operator by means of the valence quark mass dependence of the chiral condensate in partially quenched Chiral Perturbation Theory (pqChPT). This is the extension of standard ChPT with an additional valence quark. Its mass is required to probe the Dirac spectrum. We have considered two different domains of applicability: the ergodic domain with $m_{v} \ll F^{2} / L^{2} \Sigma_{0}$ and the diffusive domain with $F^{2} / L^{2} \Sigma_{0} \ll m_{v} \ll \Lambda_{Q C D}$. The energy scale $F^{2} / L^{2} \Sigma_{0}$ is the equivalent of the Thouless energy in mesoscopic physics. In the ergodic domain the partition function is dominated by the zero momentum modes and can be reduced to chiral Random Matrix Theory (chRMT). In particular, we have shown this for the microscopic spectral density, the valence quark mass dependence of the chiral condensate and the asymptotic tail of the two-point spectral correlation function. We have found that a noncompact superunitary measure is required to directly reproduce the correct valence quark mass dependence. These results strongly suggest that the zero momentum component of the pqChPT partition function is equivalent to the chRMT partition function.

The diffusive regime is the realm of Chiral Perturbation Theory. We have reproduced the Smilga-Stern expression for the slope of the spectral density of the QCD Dirac operator and have calculated its first order correction in the quark mass. We have shown that these results are consistent with standard ChPT. For nonzero sea quark masses the spectral density diverges logarithmically with a coefficient that is proportional to the screened topological susceptibility. 
We have studied the transition from chRMT to pqChPT in the domain where both are applicable and have found that both theories give the same valence quark mass dependence of the chiral condensate in this domain. The Thouless energy can thus be derived from the finite volume scalar propagator.

If pqChPT provides an accurate description of the QCD partition function we have shown that up to the Thouless energy the Dirac spectrum can be described by chRMT. Agreement with lattice QCD simulations suggests that this is indeed the case.

Other scenarios are possible. For example, the proof of the Vafa-Witten theorem [75] is not valid for imaginary quark masses. Therefore, breaking of "isospin" symmetry between the sea quark sector and the valence quark sector cannot be excluded. Related to this is the possibility that the pion decay constant is different for the sea quark channels and the valence quark channels. This might restrict the domain of validity of chRMT. However, universality arguments suggest that such scenarios are unlikely. Ultimately, these and other dynamical questions have to be answered by means of lattice QCD simulations.

\section{Acknowledgements}

This work was partially supported by the US DOE grant DE-FG-88ER40388. One of us (D.T.) was supported by Schweizerischer Nationalfonds. T. Guhr, A. Schäfer, A. Smilga, M. Stephanov, H.A. Weidenmüller and T. Wettig are acknowledged for useful discussions. We particularly benefitted from many useful comments by P. Damgaard.

\section{Appendix A. Calculation of the Microscopic Spectral Density for $N_{f}=1$}

In this appendix we calculate the integrals in the calculation of the microscopic spectral density from (45). We explicitly work out the integrals for $N_{f}=1$ and indicate how they can be performed for arbitrary $N_{f}$. For $N_{f}=1$ the different factors contributing to the integral are given by

$$
\begin{aligned}
B(\Lambda) & =\frac{\left(e^{i \theta_{0}}-e^{i \theta_{1}}\right)}{\left(e^{i \theta_{0}}-e^{i \theta_{2}}\right)\left(e^{i \theta_{1}}-e^{i \theta_{2}}\right)} \\
B(\cos \theta) & =\frac{\left(\cos \theta_{0}-\cos \theta_{1}\right)}{\left(\cos \theta_{0}-\cos \theta_{2}\right)\left(\cos \theta_{1}-\cos \theta_{2}\right)}, \\
B\left(M_{k}\right) & =\frac{\left(m_{v}-m_{s}\right)}{2 J \Sigma_{0} V\left(m_{v}-m_{s}\right)}=\frac{1}{2 J \Sigma_{0} V} .
\end{aligned}
$$


In the chiral limit $m_{s} \rightarrow 0$, the determinant can be written as

$$
\operatorname{det} e^{M_{k} V \Sigma_{0} \cos \theta_{l}}=e^{m_{v} V \Sigma_{0} \cos \theta_{0}}-e^{m_{v} V \Sigma_{0} \cos \theta_{1}} .
$$

Combining all factors we find

$$
\left.\frac{1}{V} \partial_{J} Z^{C}(J)\right|_{J=0}=\frac{\Sigma_{0}}{8 \pi^{3}} \int d \theta_{0} d \theta_{1} d \theta_{2} \frac{\left|e^{i \theta_{0}}-e^{i \theta_{1}}\right|^{2}\left(\cos \theta_{0}-\cos \theta_{2}\right)\left(\cos \theta_{1}-\cos \theta_{2}\right)}{\left|e^{i \theta_{0}}-e^{i \theta_{2}}\right|^{2}\left|e^{i \theta_{1}}-e^{i \theta_{2}}\right|^{2}\left(\cos \theta_{0}-\cos \theta_{1}\right)} e^{m_{v} V \Sigma_{0}\left(\cos \theta_{0}-\cos \theta_{2}\right)},
$$

where we have used the anti-symmetry of the measure in $\theta_{0}$ and $\theta_{1}$.

We first perform the integral over $\theta_{1}$

$$
I_{1} \equiv \int d \theta_{1} \frac{\left|e^{i \theta_{0}}-e^{i \theta_{1}}\right|^{2}\left(\cos \theta_{1}-\cos \theta_{2}\right)}{\left(\cos \theta_{0}-\cos \theta_{1}\right)\left|e^{i \theta_{1}}-e^{i \theta_{2}}\right|^{2}} .
$$

The integrand can be simplified with the help of the identity (38) (for $s=i \phi$ ) resulting in

$$
I_{1}=\int d \theta_{1} \frac{\left(e^{-i \theta_{0}}-e^{-i \theta_{1}}\right)\left(1-e^{-i \theta_{1}} e^{-i \theta_{2}}\right)}{\left(1-e^{-i \theta_{0}} e^{-i \theta_{1}}\right)\left(e^{-i \theta_{1}}-e^{-i \theta_{2}}\right)} .
$$

To evaluate the integral we add opposite infinitesimal imaginary increments to $\theta_{0}$ and $\theta_{2}$ and take the limit after the integration. For definiteness, let us add a negative imaginary increment to $\theta_{0}$. Then we can expand the appropriate terms in a geometric series. We observe that $\theta_{1}$ only occurs as positive powers of $\exp \left(-i \theta_{1}\right)$, and only the constant term contributes. The result for the integral is thus

$$
I_{1}=-2 \pi e^{i \theta_{2}-i \theta_{0}}
$$

For the opposite choice of the imaginary increment we obtain the complex conjugate expression resulting in the same final answer,

$$
\left.\frac{1}{V} \partial_{J} Z(J)\right|_{J=0}=-\frac{\Sigma_{0}}{4 \pi^{2}} \int d \theta_{0} d \theta_{2} e^{i \theta_{2}-i \theta_{0}} \frac{\left(\cos \theta_{0}-\cos \theta_{2}\right)}{\left|e^{i \theta_{0}}-e^{i \theta_{2}}\right|^{2}} e^{x\left(\cos \theta_{0}-\cos \theta_{2}\right)},
$$

where $x=m_{v} V \Sigma_{0}$. Notice that only the real part of $I_{1}$ contributes to the integral over $\theta_{2}$ and $\theta_{0}$. It is simplest to evaluate this integral by first differentiating it with respect to $x$ and using once more the identity (38)

$$
\begin{aligned}
\partial_{x} \Sigma(x) & =-\frac{\Sigma_{0}}{16 \pi^{2}} \int d \theta_{2} d \theta_{0}\left(1-e^{-i \theta_{0}-i \theta_{2}}\right)\left(1-e^{+i \theta_{0}+i \theta_{2}}\right) e^{i \theta_{2}-i \theta_{0}} e^{x\left(\cos \theta_{0}-\cos \theta_{2}\right)}, \\
& =\frac{\Sigma_{0}}{2}\left[J_{1}(-i x) J_{1}(-i x)+J_{0}(-i x) J_{2}(-i x)\right], \\
& =\frac{\Sigma_{0}}{2} \partial_{x}\left[x J_{1}^{2}(-i x)-x J_{0}(-i x) J_{2}(-i x)\right] .
\end{aligned}
$$


This equation can be integrated trivially with an integration constant that is determined by the asymptotic limit for the spectral density. The result for the microscopic spectral density is obtained by putting $m_{v}=i \lambda$ and taking the microscopic limit according to (田) (with $u=\lambda V \Sigma_{0}$ ),

$$
\rho_{S}(u)=\frac{u}{2}\left[J_{1}^{2}(u)-J_{0}(u) J_{2}(u)\right]
$$

in complete agreement with the chRMT result for $N_{f}=1$.

For an arbitrary number of flavors $N_{f}$, we systematically use the symmetries of the integrand. First, the determinant $\operatorname{det}_{0 \leq k, l \leq N_{f}}\left[\exp \left(m_{k} \cos \theta_{l}\right)\right]$ appearing in (45) and the Berezinian $B\left(\cos \theta_{i}\right)$ are both antisymmetric in $\theta_{i} \leftrightarrow \theta_{j}$, for $i \neq j$ and $i, j=0, \cdots, N_{f}$. Therefore the whole expression can be simplified to:

$$
\partial_{J} Z_{p q}^{0} \sim \int d \theta_{0} d \theta_{N_{f}+1} \frac{\cos \theta_{0}-\cos \theta_{N_{f}+1}}{\left|e^{i \theta_{0}}-e^{i \theta_{N_{f}+1}}\right|^{2}} e^{x\left(\cos \theta_{0}-\cos \theta_{N_{f}+1}\right)} \tau_{N_{f}}\left(\theta_{N_{f}+1}, \theta_{0}\right),
$$

where

$$
\tau_{N_{f}}\left(\theta_{N_{f}+1}, \theta_{0}\right)=\int \prod_{k=1}^{N_{f}} d \theta_{k}\left|\Delta\left(e^{i \theta_{k}}\right)\right|^{2} \omega\left(\theta_{N_{f}+1}, \theta_{k}\right) \omega\left(-\theta_{0}, \theta_{k}\right) .
$$

and $\omega(\theta, \phi)$ is defined by

$$
\omega(\theta, \phi)=\frac{\cos \theta-\cos \phi}{\left|e^{i \theta}-e^{i \phi}\right|^{2}}
$$

Considering again $\partial_{x} \Sigma(x)$ and using the fact that $\left|\Delta\left(e^{i \theta_{k}}\right)\right|^{2}$ is the sum of 1 plus terms $\exp \left(i \sum_{k} n_{k} \theta_{k}\right)$ with $n_{k}$ finite and $\sum_{k} n_{k}=0$ [47], we can show that the only term in $\tau_{N_{f}}\left(\theta_{N_{f}+1}, \theta_{0}\right)$ contributing to the integral is proportional to $\exp \left[i N_{f}\left(\theta_{0}-\theta_{N_{f}+1}\right)\right]$. Notice, that the term proportional to $\sin \left[N_{f}\left(\theta_{N_{f}+1}-\theta_{0}\right)\right]$ does not contribute to the integral. To obtain the final result for the microscopic spectral density we again use the symmetry properties of the integrand with respect to the exchange and translations of the $\theta_{k}$ variables.

\section{Appendix B}

In this appendix we calculate the Berezinian corresponding to the diagonalization of a superunitary matrix. The calculation is analogous to the case of a super-Hermitean matrix [65, 66]. We can decompose the superunitary matrix $U$ into its eigenvalues $\Lambda$ and another superunitary matrix $V$ by $U=V \Lambda V^{\dagger}$. Since $U$ is superunitary its eigenvalues are just phases, i.e. $\Lambda=\operatorname{diag}\left(e^{i \theta_{k}}\right)$. The relevant length element is

$$
|d s|^{2}=\operatorname{Str} d U d U^{\dagger}=\operatorname{Str}\left(d \Lambda d \Lambda^{\dagger}\right)+\operatorname{Str}\left(\left[V^{\dagger} d V, \Lambda\right]\left[V^{\dagger} d V, \Lambda\right]^{\dagger}\right) .
$$


Since we are only interested in the $\Lambda$ dependence of the Berezinian we can use the parametrization $V=e^{i H}$ so that $V^{\dagger} d V=i d H$. We then have

$$
|d s|^{2}=\sum_{i} \epsilon(i) d \Lambda_{i} d \Lambda_{i}^{*}+\sum_{i, j} \epsilon(i) \epsilon(j)\left|\Lambda_{i}-\Lambda_{j}\right|^{2} d H_{i, j} d H_{i, j}^{*}
$$

where $\epsilon(i)=1(-1)$ if the $\Lambda_{i}$ corresponds to a fermion (boson). If we write this in the form $|d s|^{2}=g_{a, b} d z_{a} d z_{b}^{*}$ then the Berezinian is given by $\sqrt{\operatorname{Sdet}(g)}$,

$$
|B(\Lambda)|^{2}=\frac{\prod_{i>j}\left|\Lambda_{i}^{f}-\Lambda_{j}^{f}\right|^{2} \prod_{i>j}\left|\Lambda_{i}^{b}-\Lambda_{j}^{b}\right|^{2}}{\prod_{i, j}\left|\Lambda_{i}^{f}-\Lambda_{j}^{b}\right|^{2}} .
$$

\section{References}

[1] C. DeTar, Quark-gluon plasma in numerical simulations of QCD, in Quark gluon plasma 2, R. Hwa ed., World Scientific 1995.

[2] A. Ukawa, Nucl. Phys. Proc. Suppl. 53 (1997) 106.

[3] A.V. Smilga, Phys. Rep. 291, (1997) 1.

[4] T. Banks and A. Casher, Nucl. Phys. B169 (1980). 103

[5] E.V. Shuryak and J.J.M. Verbaarschot, Nucl. Phys. A560 (1993) 306.

[6] J.J.M. Verbaarschot, Nucl. Phys. B427 (1994) 434.

[7] M.E. Berbenni-Bitsch, S. Meyer, A. Schäfer, J.J.M. Verbaarschot and T. Wettig, Phys. Rev. Lett. 80 (1998) 1146.

[8] M.E. Berbenni-Bitsch, M. Gockeler, T. Guhr, A.D. Jackson, J.Z. Ma, S. Meyer, A. Schäfer, H.A. Weidenmüller, T. Wettig and T. Wilke, Crossover to nonuniversal fluctuations in lattice gauge theory, hep-ph/9804439.

[9] T. Guhr, J.Z. Ma, S. Meyer and T. Wilke, Statistical analysis and the equivalent of a Thouless energy in lattice QCD Dirac spectra, hep-lat/9806003.

[10] M.E. Berbenni-Bitsch, S. Meyer and T. Wettig, hep-lat/9804030.

[11] M.A. Halasz and J.J.M. Verbaarschot, Phys. Rev. Lett. 74 (1995) 3920; M.A. Halasz, T. Kalkreuter and J.J.M. Verbaarschot, Nucl. Phys. Proc. Suppl. 53 (1997) 266.

[12] J.Z. Ma, T. Guhr and T. Wettig, Eur. Phys. J. A2 (1998) 87.

[13] R. Pullirsch, K. Rabitsch, T. Wettig and H. Markum, hep-ph/9803285. 
[14] G. Akemann, P. Damgaard, U. Magnea and S. Nishigaki, Nucl. Phys. B 487[FS] (1997) 721.

[15] E. Brézin, S. Hikami and A. Zee, Nucl. Phys. B464 (1996) 411.

[16] T. Guhr and T. Wettig, Nucl. Phys. B506 (1997) 589.

[17] A.D. Jackson, M.K. Sener and J.J.M. Verbaarschot, Nucl. Phys. B479 (1996) 707.

[18] A.D. Jackson, M.K. Sener and J.J.M. Verbaarschot, Nucl. Phys. B506 (1997) 612.

[19] K. Splittorff and A.D. Jackson, hep-lat/9805018.

[20] S.M. Nishigaki, P.H. Damgaard and T. Wettig, hep-th/9803007.

[21] M.K. Sener and J.J.M. Verbaarschot, hep-th/9801042.

[22] D. Callaway and A. Rahman, Phys. Rev. Lett. 49 (1982) 613; J. Polony and H.W. Wyld, Phys. Rev. Lett. 51 (1983) 2257; J. Kogut, J. Polony, J. Shigemitsu, D.K. Sinclair and H.W. Wyld, Phys. Rev. Lett. 53 (1984) 644.

[23] O. Bohigas, M. Giannoni, Lecture notes in Physics 209 (1984) 1; O. Bohigas, M. Giannoni and C. Schmit, Phys. Rev. Lett. 52 (1984) 1.

[24] J. Stern, hep-ph/9801282.

[25] T. Guhr, A. Müller-Groeling and H.A. Weidenmüller, Phys. Rept. 299 (1998) 189.

[26] C.W.J. Beenakker, Rev. Mod. Phys. 69 (1997) 731.

[27] G. Montambaux, in Quantum Fluctuations, Les Houches, Session LXIII, E. Giacobino, S. Reynaud and J. Zinn-Justin, eds., Elsevier Science, 1995, condmat/9602071.

[28] B.L. Altshuler, I.Kh. Zharekeshev, S.A. Kotochigova and B.I. Shklovskii, Zh. Eksp. Teor. Fiz. 94 (1988) 343.

[29] H.A. Weidenmüller, Nucl. Phys. A 518 (1990) 1.

[30] T. Guhr and A. Mueller-Groeling, cond-mat/9702113, J. Math. Phys. (in press).

[31] N. Argaman, Y. Imry and U. Smilansky, Phys. Rev. B47 (1993) 4440.

[32] V.E. Kravtsov, in Correlated Fermions and Transport in Mesoscopic Systems, Les Arcs, 1996, cond/mat9603166.

[33] A. Altland and Y. Gefen, Phys. Rev. Lett. 71 (1993) 3339. 
[34] D. Braun and G. Montambaux, Phys. Rev. 52 (1995) 13903.

[35] A.G. Aronov and A.D. Mirlin, Phys. Rev. B51 (1995) 6131.

[36] Y.V. Fyodorov and A.D. Mirlin, Phys. Rev. B51 (1995) 13403.

[37] V.E. Kravtsov, I.V. Lerner, B.L. Altshuler and A.G. Aronov, Phys. Rev. Lett. 72 (1994) 888.

[38] A. Altland, Y. Gefen and G. Montambaux, Phys. Rev. lett. 76 (1996) 1130.

[39] J.T. Chalker, V.E. Kravtsov, I.V. Lerner, JETP Lett. 64 (1996) 386.

[40] E. Shuryak, Nucl. Phys. B302 (1988) 599; D. Diakonov, Talk given at International School of Physics, 'Enrico Fermi', Course 80: Selected Topics in Nonperturbative QCD, Varenna, 1995, hep-ph/9602375.

[41] J.C. Osborn and J.J.M. Verbaarschot, Phys. Rev. Lett. (in press) (1998).

[42] R. Janik, G. Papp, M. Nowak and I.Zahed, hep-ph/9803289.

[43] J.C. Osborn and J.J.M. Verbaarschot, Nucl. Phys. B (in press) (1998), hepph/9803419.

[44] A. Morel, J. Physique 48 (1987) 1111.

[45] C. Bernard and M. Golterman, Phys. Rev. D49 (1994) 486; C. Bernard and M. Golterman, hep-lat/9311070.

[46] J. Gasser and H. Leutwyler, Phys. Lett. 188B(1987) 477; Nucl. Phys. B307 (1988) 763.

[47] H. Leutwyler and A. Smilga, Phys. Rev. D46 (1992) 5607.

[48] J. Verbaarschot, Phys. Rev. Lett. 72 (1994) 2531; Phys. Lett. B329 (1994) 351; Nucl. Phys. B426[FS] (1994) 559.

[49] J.J.M. Verbaarschot, Lectures given at NATO Advanced Study Institute on Confinement, Duality and Nonperturbative Aspects of QCD, Cambridge, 1997, hepth/9710114.

[50] S. Chandrasekharan, Nucl. Phys. Proc. Suppl. 42 (1995) 475; S. Chandrasekharan and N. Christ, Nucl. Phys. Proc. Suppl. 42 (1996) 527; N. Christ, Nucl. Phys. B (Proc. Suppl.) 53 (1997) 253.

[51] J.J.M. Verbaarschot, Phys. Lett. B368 (1996) 137. 
[52] J.J.M. Verbaarschot, in Nonperturbative Approaches to Quantum Chromodynamics, D. Diakonov, ed., Gatchina 1995.

[53] A.V. Andreev, B.D. Simons, and N. Taniguchi, Nucl. Phys B432 [FS] (1994) 487.

[54] J.C. Osborn, D. Toublan and J.J.M. Verbaarschot, in preparation.

[55] M.F.L. Golterman and K.C. Leung, hep-lat/9711033; M.F.L. Golterman, Acta Phys. Polon. B25 (1994).

[56] A. Smilga and J. Stern, Phys. Lett. B318 (1993) 531.

[57] P.H. Damgaard, Phys. Lett. B424 (1998) 322; G. Akemann and P.H. Damgaard, hep-th/9802174; G. Akemann and P.H. Damgaard, hep-th/9801133.

[58] J. Gasser and H. Leutwyler, Ann. Phys. 158, 142 (1984); J. Gasser and H. Leutwyler, Nucl. Phys. B250, 465 (1985).

[59] S. Sharpe, Phys. Rev. D 56 (1997) 7052.

[60] C. Bernard and M. Golterman, Phys. Rev. D46 (1992) 853; M. Golterman, heplat/9411005, Chiral Perturbation Theory and the quenched approximation of QCD; S. Sharpe, Phys. Rev. D46 (1992) 3146.

[61] G. Colangelo and E. Pallante, Nucl. Phys. B520 (1998) 433.

[62] J. Verbaarschot, H. Weidenmüller, and M. Zirnbauer, Phys. Rep. 129 (1985) 367.

[63] M. Zirnbauer, J. Math. Phys. 37 (1996) 4986.

[64] P. Damgaard, J. Osborn, D. Toublan and J. Verbaarschot, in preparation.

[65] T. Guhr, J. Math. Phys. 32 (1991) 336.

[66] J. Alfaro, R. Medina and L.F. Urrutia, J. Math. Phys. 36 (1995) 3085.

[67] T. Guhr and T. Wettig, J. Math. Phys. 37 (1996) 6395.

[68] K.B. Efetov, Adv. Phys. 32 (1983) 53.

[69] F. Wegner, private communication, 1983.

[70] M.R. Zirnbauer, Nucl. Phys. B 265 [FS15] (1986) 375.

[71] M. J. Rothstein, Trans. Am. Math. Soc. 299, 387 (1987).

[72] J. Verbaarschot and I. Zahed, Phys. Rev. Lett. 70, 3852 (1993). 
[73] P. Hasenfratz and H. Leutwyler, Nucl. Phys. B343 (1990) 241.

[74] M. Shifman, A. Vainshtein and V. Zakharov, Nucl. Phys. B166 (1980) 493.

[75] C. Vafa and E. Witten, Nucl. Phys. B234 (1984) 173. 